\title{
Le bruit laisse son empreinte
}

\section{Ottilia Lütolf Elsener}

Médecin spécialiste en angiologie et en médecine interne, membre de l'association des Médecins en faveur de l'Environnement (MfE), membre de la Commission fédérale pour la lutte contre le bruit (CFLB), groupe de travail "Journée contre le bruit», membre de la FMH des BAFU «Auswirkungen des Verkehrslärms auf die Gesundheit», Bern und Altdorf, 2014

2 Lärmwirkungsstudie

NORAH, Bochum, 2015. Christensen JS, Hiortebjerg D, Raaschou-Nielsen O, Ketzel M, Sørensen TI, Sørensen M. Pregnancy and childhood exposure to residential traffic noise and overweight at 7 years of age. Environment International. 2016;94:170-6.

L’article a été élaboré en collaboration avec Nadia Sauter Oes, médecin spécialiste FMH en pédiatrie, Winterthour, et Andrea Kaufmann coordinatrice de la "Journée contre le bruit", Lucerne.
1 Ecoplanstudie im Auftrag

Qu'il s'agisse de troubles de sommeil, d'hypertension artérielle ou de lésions auditives - le bruit peut rendre les gens malade. Les adultes ne sont pas les seuls à souffrir du bruit, les enfants sont aussi concernés. Les effets d'une exposition au bruit excessif ne sont pas uniquement de nature physique, une souffrance psychologique ou une diminution des performances cognitives peuvent également se manifester.

Le bruit est un facteur de stress. Notre oreille est un organe extrêmement sensible, adaptée au fond sonore d'une nature aujourd'hui disparue. Les bruits élevés et inhabituels étaient perçus, autrefois, comme un signal de danger auquel le corps humain, en état d'alerte maximal, réagissait en se préparant à fuir ou à combattre. Depuis, notre environnement sonore s'est complètement modifié: Une multitude de bruits nous submerge en permanence. Notre organisme continue, cependant, de réagir à des bruits forts et gênants comme il le faisait dans les temps anciens. Il sécrète des hormones en grande quantité, l'adrénaline et la noradrénaline et il puise dans ses réserves d'énergie. A cela s'ajoute une libération de cortisol en cas d'augmentation de l'intensité sonore et de la durée d'exposition - ces réactions impactent notre bien-être et notre santé. Il n'y a pas d'habituation de l'organisme en matière de bruit.

\section{Les enfants aussi souffrent du bruit}

Les effets du bruit sur les enfants font rarement l'objet de débats médiatisés, et pourtant des études démontrent que l'exposition chronique au bruit peut

\section{Journée contre le bruit}

Le 26 avril 2017 aura lieu la journée contre le bruit. La Suisse participe à cette journée d'action depuis 2005. Chaque année, un autre volet de la problématique du bruit est mis en lumière. Cette année, la journée contre le bruit est placée sous la devise "Le calme, un moteur» et attirera notre attention sur les effets du bruit sur les enfants. Le bruit dérange et peut rendre malade. Pour un développement sain, les enfants ont besoin d'un environnement sain. Le calme en est effectivement un élément moteur. Afin de sensibiliser les parents et les personnes concernées par ce thème, nous mettons à votre service un dépliant attractif qui, une fois découpé, devient un accroche-porte. Ces dépliants peuvent être laissés à disposition dans votre cabinet. Commandez-les encore aujourd'hui gratuitement sous www.bruit.ch/ enfants et collaborez à la protection des enfants du bruit excessif et de ses effets nocifs. La "Journée contre le bruit» est mise sur pied par le Cercle Bruit, la Société Suisse d'Acoustique, la ligue Suisse contre le Bruit et Médecins en faveur de l'environnement. Elle bénéficie, en outre, du soutien de l'Office fédéral de I'environnement (OFEV) et de l'Office fédéral de la santé publique (OFSP). Vous trouverez des informations plus détaillées sur le site www. bruit.ch/enfants. provoquer, chez les enfants, des troubles persistants physiques et mentales. Nous devons être conscients que les enfants sont moins capables que les adultes d'influencer leur environnement sonore. Ils sont fréquemment exposés au bruit sans pouvoir y échapper. Leurs stratégies d'adaptation (dites coping), correspondant à leur âge, ne les protègent pas des effets sanitaires du bruit. De plus, les enfants ont une autre perception du danger et ne savent pas reconnaître une situation dangereuse liée notamment au bruit.

\section{Troubles de l'apprentissage}

Selon l'étude Ecoplanstudie ${ }^{1}$ (2014), 17,5 pourcent de tous les enfants, âgés de 7 à 19 ans, développent des troubles de l'apprentissage lorsqu'ils sont exposés au bruit des transports. Le bruit altère la mémoire chez l'enfant. Il en résulte une diminution de la concentration et de la capacité d'attention ce qui ralentit le processus d'apprentissage par rapport aux enfants non exposés au bruit.

Les résultats de l'étude NORAH ${ }^{2}$ ont été publiés en octobre 2015. Cette étude explore les effets du bruit de la circulation sur la qualité de vie des enfants, sur leur santé et leur développement, dans la région du RhinMain. Dans les zones fortement touchées par le bruit généré par les avions, les enfants apprennent à lire plus lentement que les enfants résidant dans des endroits tranquilles. Une augmentation du niveau sonore de 10 décibels prolonge l'apprentissage de la lecture d'un mois.

Une déficience des performances cognitives de l'enfant, due à une exposition à un fort niveau sonore, impactera sa vie d'adulte. Il éprouvera des difficultés dans ses études supérieures car la capacité de lecture, d'écriture et d'attention a été affectée.

\section{Perturbations du sommeil et troubles du comportement}

Le bruit n'a pas seulement des effets néfastes sur les aptitudes cognitives de l'enfant. Notamment, les troubles du sommeil et le stress occasionnés par le bruit de la circulation peuvent entraîner des problèmes d'obésité et de diabète. ${ }^{3}$ Les réactions de réveil, au cours de la nuit, se produisent souvent de manière inconsciente, 
c'est pourquoi, le matin, les enfants ne savent pas que leur sommeil a été perturbé. Pourtant, le repos de la nuit, spécialement pour les enfants, est d'une importance capitale. Les troubles du sommeil passent trop souvent inaperçus, et compromettent, à long terme, un développement sain. Les enfants dont la chambre donne sur une rue à grande circulation, ont aussi tendance à avoir une pression artérielle légèrement plus élevée. Lorsque l'organisme souffre d'hypertension artérielle pendant de longues années, le risque de présenter des maladies cardio-vasculaires à l'âge adulte est plus fréquent. L'hyperactivité et certains troubles du comportement ont été également attribués à une exposition prolongée au bruit de la circulation. ${ }^{4}$

Même dans des zones rurales, supposées plus calmes, les enfants sont aussi affectés par le bruit. Une étude, ${ }^{5}$ concernant 1280 élèves de l'école populaire du Tyrol, a démontré de manière significative que des symptômes tels que l'anxiété, la dépression ou les troubles du comportement allaient de pair avec l'élévation des nuisances sonores générées par les transports. Il semble que dans les vallées alpines, les nuisances sonores sont moins bien supportées car l'organisme humain réagit par rapport au bruit ambiant. Les pics sonores nocturnes occasionnés par le trafic ferroviaire ont été particulièrement déterminants dans cette étude.

\section{Impact du bruit sur les nourrissons}

4 Hjortebjerg D, Andersen AM, Christensen JS, Ketzel M, Raaschou-Nielsen O Sunyer J, Julvez J, Forns J, Sørensen M. Exposure to road traffic noise and behavioral problems in 7-year-old children: a cohort study. Environ Health Perspect. 2016; 124:228-34

5 Lercher P, Evans GW, Meis M, Kofler WW. Ambient neighbourhood noise and children's mental health Occupational and Environmental Medicine. 2002;59:380-6.

6 Chang $\mathrm{E}$ et al. Environmental Noise Retards Auditory Cortical Development. Science. 2003 300:498-502.

Correspondance:
Andrea Kaufmann
dipl. Geografin
Projektleiterin
Obere Isenegg 2
CH-9555 Tobel
Tél. 0434996477
andrea.kaufmann[at]
umsicht.ch

Correspondance: Andrea Kaufmann dipl. Geografin Projektleiterin Obere Isenegg 2 CH-9555 Tobel umsicht.ch
Le bruit marque le cerveau. Les chercheurs américains Edward Chang et Michael Merzenich ${ }^{6}$ ont exposé des ratons dès la naissance pendant plusieurs mois à un son de niveau sonore comparable à un environnement sonore habituel. Bien que cela ne cause aucun dommage auditif chez les animaux, il a été néanmoins observé un retard de développement du cortex auditif où se forment, au cours du premier mois de vie, des associations de neurones qui réagissent de manière sélective à des sons et fréquences particuliers. Chez les rats qui ont été exposés au bruit en permanence, ce processus de maturation fait défaut pendant des mois. Il est concevable que des circonstances similaires laissent aussi une marque durable chez les bébés. Une exposition à un fond sonore élevé peut donc affecter le développement du cerveau et ainsi nuire à l'audition et à la capacité de langage. D’autre part, les tout-petits apprennent avec plus de difficultés à parler lorsque la radio et la télévision sont allumées en fond sonore, en permanence. On a aussi tendance à moins leur adresser directement la parole. Il est très difficile, pour les enfants, de discerner, du bruit ambiant, les mots qui leur sont adressés - et pourtant les enfants apprennent à parler en écoutant.

\section{Troubles auditifs}

Diverses recherches menées auprès des enfants et des adolescents montrent qu'une exposition à des niveaux sonores de 100 à $120 \mathrm{~dB}(\mathrm{~A})$, de quelques minutes à plusieurs heures, peut causer un traumatisme sonore aigu. Une telle exposition au bruit entraîne aussi bien des dommages métaboliques que mécaniques au niveau des cellules auditives situées dans l'oreille interne. Dans la plupart des cas, les troubles auditives se développent de manière insidieuse pendant l'enfance et l'adolescence. Néanmoins, exposé à une pression acoustique de $130 \mathrm{~dB}(\mathrm{~A})$, quelques secondes seulement suffisent à endommager l'audition. Chez le nouveauné, jusqu'à l'âge de huit mois, le développement du nerf auditif, qui relie la cochlée au cerveau, n'est pas arrivé à maturité. Une exposition d'une durée de 15 à 30 minutes, à une fanfare de rue, par exemple, peut engendrer, à cet âge, des atteintes irréversibles de l'ouïe. Les adultes et les enfants plus âgés, dans cette situation, peuvent se boucher les oreilles pour se protéger ou s'éloigner de la source des bruits. Les jeunes enfants et les nourrissons n'en sont pas capables.

En plus, à cause des particularités du conduit auditif chez les tout-petits, à intensité égale, le jeune enfant perçoit le bruit nettement plus fort que l'adulte. C'est pourquoi, une exposition au bruit, même de courte durée, peut entraîner des lésions auditives. Jusqu'à l'âge de 5 ans, l'ouie chez l'enfant est particulièrement sensible.

\section{Un développement sain}

Pour que les enfants puissent se développer sainement, sans les conséquences négatives à long terme du bruit, ils ont besoin d'un environnement sain. A l'avenir, il conviendra d'accorder une attention accrue aux nuisances sonores. En effet, l'exposition excessive au bruit peut avoir sur les nourrissons et les enfants des répercussions à vie. En 1910, Robert Koch, bactériologiste, le savait déjà: «Un jour, l'être humain devra combattre le bruit aussi inexorablement que le choléra et la peste.» Sa prophétie nous a déjà rattrapés.

\section{Bruits et niveau sonore}

Le bruit est un son indésirable. Le niveau sonore est donné en décibel (dB). L'échelle logarithmique des décibels est de $0 \mathrm{~dB}$ (seuil d'audibilité) à $130 \mathrm{~dB}$ (seuil de douleur) dans la gamme de volume, à intervalles réguliers; $10 \mathrm{~dB}$ : le tic-tac d'une montre-bracelet, 40 $\mathrm{dB}$ : musique douce, $60 \mathrm{~dB}$ : une conversation tout à fait normale, 90 $\mathrm{dB}$ : un camion, $120 \mathrm{~dB}$ : un marteau piqueur pneumatique.

L'être humain peut percevoir, sous certaines conditions, une variation de $1 \mathrm{~dB}$ du niveau sonore.

Une variation de $10 \mathrm{~dB}$ équivaut, à peu près, à une multiplication ou à une division par deux du niveau sonore subjectivement perçu. 